\title{
A silent period in orbicularis oculi muscles of humans
}

\author{
JEROME N SANES AND JAMES R ISON \\ From the Reflex Laboratory, Department of Psychology, University of Rochester, Rochester, New York
}

SUMMARY Surface electromyographic activity was recorded bilaterally from orbicularis oculi muscles when subjects relaxed and contracted eyelid muscles. Cutaneous reflex responses were evoked during both the relaxed and contraction states. Following reflex elicitation periods of muscle silence in orbicularis oculi were observed for about 10 to $15 \mathrm{~ms}$ after the ipsilateral R1 response and for up to $100 \mathrm{~ms}$ after the bilateral $\mathrm{R} 2$ responses. Reflex responses appeared to be enhanced when elicited during contractions. Possible physiological mechanisms are discussed regarding the presence of silent periods in a motor system that is presumably devoid of spindles, Golgi tendon organs, and Renshaw-like interneurons.

When a reflexive response is elicited in a muscle which is already in a state of sustained contraction, then the additional contraction produced by the reflex is typically followed by a temporary ciessation of activity. This is the "silent period," and its explanation, of which several are current, relies extensively on the physiology of segmental interconnections and on the intrinsic properties of motoneurons. Thus, it may depend on the withdrawal of excitatory spindle input by unloading the muscle receptor, by autogenic inhibition produced by Golgi tendon organs, by activation of Renshaw cells and their recurrent inhibition, by hyperpolarisation following discharge, and the ensuing refractory period, or by any combination of these effects. ${ }^{361724}$ Additionally, there has been some suggestion that cutaneous afferents and long propriospinal pathways may mediate muscle silence after voluntary or reflex contractions. ${ }^{1112}$

Silent periods are most often studied in load bearing muscles where all of these segmental mechanisms may potentially be engaged. The search for a silent period in the eyelid musculature, in the palpebral portion of orbicularis oculi, is especially interesting because of the absence there of many of these mechanisms. There are apparently no muscle spindles or Golgi tendon organs associated with the orbicularis oculi muscle, nor are interneurons seen in the facial nucleus. ${ }^{2} 27$ Thus, there seems little likeli-

Address for reprint requests: Dr Jerome $N$ Sanes, Laboratory of Neurophysiology, National Institute of Mental Health, Building 36, Room 2D-10, Bethesda, Maryland 20205 U.S.A.

Accepted 21 February 1980 hood that autogenic or recurrent inhibition could produce muscle silence following activation of the eyeblink reflex arc. In line with the suggested importance of these mechanisms, and of their absence in orbicularis oculi muscle, there are now several experiments reporting an absence of a silent period in orbicularis oculi when an eyeblink reflex is elicited during sustained voluntary eyelid muscle contractions. ${ }^{19} 2425$ However, in the course of several experiments in our laboratory, concerned with the interactions of reflex responding and muscle activity conditioned by instructions $^{21}$ or Pavlovian conditioning, ${ }^{7}$ we have observed prominent periods of electromyographic (EMG) silence following the reflexive bursts of the cutaneous eyeblink reflex. These unsystematic and somewhat unexpected findings suggested that the phenomenon should be subjected to a more orderly study.

The investigation of reflexive activity in orbicularis oculi in this connection may be additionally informative because EMG analysis shows that the eyeblink reflex is composed of at least two separate events ${ }^{1023}$ that are known to have different anatomical substrates. ${ }^{5} 18$ If, as our preliminary observations suggest, there is a silent period in orbicularis oculi muscles following its reflexive activation, there is some further interest in determining whether each component of the reflex has a silent period associated with it and whether silent periods are unilateral or consensual.

\section{Methods}

Experiments were performed in an electrically isolated and sound attenuated chamber on five 
human subjects (23 to 32 years of age) who were without any observable neurological disorders. The experimenter and the control apparatus were outside this room, and thus the subject never received any inadvertent information concerning the occurrence of the stimulus conditions. They were monitored over a closed-circuit TV system and a sound intercom. Subjects were prepared for surface stimulation and recording with Beckman miniature electrodes using standard methods. Two stimulation electrodes were fixed with adhesive collars on the forehead over the supraorbital branch of the trigeminal nerve. Pairs of recording electrodes were attached to the skin at each lateral canthus and just ventromedial to this site over the inferior portions of the orbicularis oculi muscles. Intensity of the shock stimulus that evoked eyeblink reflexes was titrated for each subject so that elicited $\mathrm{R} 1$ responses had an amplitude at least two times greater than electrical noise at rest. This current varied from 7.0 to $22.0 \mathrm{~mA}$ but had a constant duration of $.5 \mathrm{~ms}$. The stimuli were never reported as painful. Reflex-eliciting stimuli were presented to all subjects when they were relaxed and when they were contracted. In the former case, the eyelids were open whereas during contraction the lids tended to be closed. Subjects were instructed to "strongly contract" both orbicularis oculi muscles simultaneously. Sometimes a "moderate" contraction was required. For four of the subjects contraction periods were alternated with periods of muscle relaxation and for one subject the contraction was maintained until all trials were delivered. These four subjects contracted and relaxed eyelid muscles at their own pace. Subjects were also instructed to maintain contractions for a short while (two to five s) after delivery of the test stimulus. On the average, trials were presented every $15 \mathrm{~s}$ (range 5 to $30 \mathrm{~s}$ ) with very few trials delivered at the short intertrial interval. For the remaining subject ten shock trials were
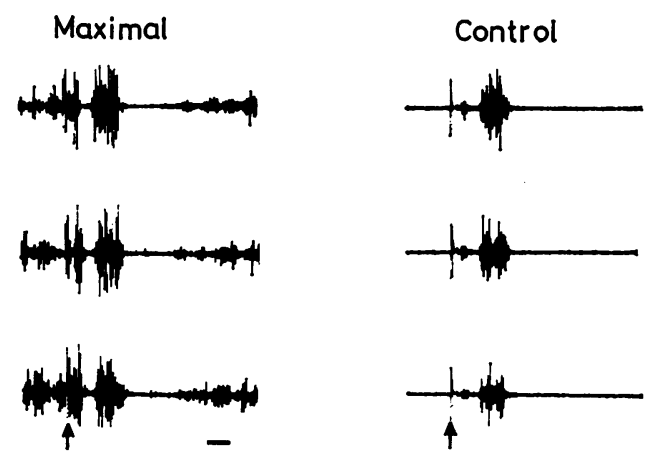

presented with an intertrial interval of 15 to $20 \mathrm{~s}$ during maintained muscle contractions. Five trials each were taken before and after onset of the prolonged contraction with an intertrial interval of 15 to $20 \mathrm{~s}$. In all cases reflex eliciting stimuli were delivered unpredictably by the experimenter from outside the chamber during muscle contractions or relaxation until at least ten of each trial type had been presented.

For numerical analyses, EMG activity from each trial was first half-wave rectified and then the peak voltages were summated, in $12 \mathrm{~ms}$ bins, for $96 \mathrm{~ms}$ prestimulus and for $240 \mathrm{~ms}$ poststimulus, starting ten ms after the shock onset. These values were summed and means were obtained. The mean values were used for the data analyses. Confidence limits were constructed about the individual subjects' baseline EMG activity and also for the group mean values. The confidence limits for the grouped data were constructed using the degrees of freedom from the repeated measures analyses of variance and a Student's $t$-table.

\section{Results}

Examples of EMG activity from one subject are depicted in fig 1 . When the eyelid muscles were relaxed the reflex stimulus elicited the unilateral $\mathrm{R} 1$ and the bilateral $\mathbf{R} 2$ responses. For this subject the signal to noise ratio of $R 1$ responses at rest was not substantial (fig 1, middle panel), but during the voluntary contraction the $R 1$ burst was typically enhanced. This was clearly evident in the traces when moderate contractions were performed (fig 1, right panel). All five subjects showed this same effect. When subjects performed near maximal contractions of orbicularis oculi muscles the size of the voluntary EMG activity sometimes obscured the $\mathbf{R} 1$ response (fig 1 , left panel).

Reflex elicitation during sustained contractions

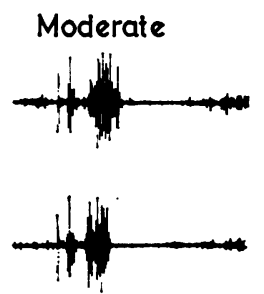

Fig 1 Electromyographic reflex activity and silent period in orbicularis oculi muscle. Ipsilateral responses from subject $J F$ during maximal contractions (left), relaxed state-labelled control (centre), and moderate contractions (right). Arrows indicate onset of reflex shock. Additional description in text.

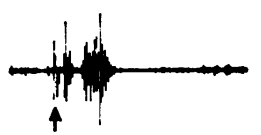



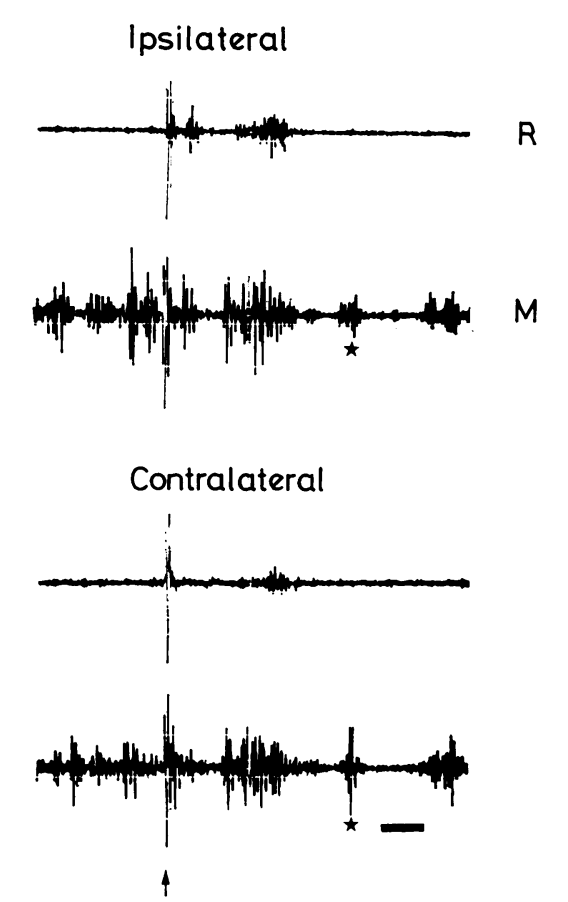

Fig 2 Ipsilateral and contralateral $E M G$ reflex responses and silent period from $L P$ during relaxed state $(R)$ and near maximal contraction $(M)$. The arrow indicates onset of reflex stimulus. Stars under both the ipsilateral and contralateral traces illustrate the appearance of $R 3$ responses when voluntary contractions of orbicularis oculi were performed.

Calibration $=20 \mathrm{~ms}$.

of the orbicularis oculi muscles resulted in a decrement of EMG activity after reflex activation of the muscle. This is illustrated in the traces of fig 1 (left panel) when this subject was required to maintain a near maximal contraction of the eyelid muscles, but it was also present during a moderate contraction (fig 1 , right panel). Note the diminution of EMG activity after the R 1 and $R 2$ responses in the ipsilateral recordings and in the contralateral recording, from another subject, (fig 2) only after the R2 response.

For the R1 response there was some withinsubject variation in the duration of the silent period over trials but little variation in the relative decrement in EMG activity during the muscle silence (fig 1, left panel). Immediately after the occurrence of the $R 1$ response there was a significant decline in EMG activity $\left(p \leqslant 0^{\wedge} 1\right)$ that varied for the group of subjects from 45 to
Table Relative EMG activities from orbicularis muscles

\begin{tabular}{|c|c|c|c|c|c|c|c|}
\hline \multirow[b]{3}{*}{$\mathrm{JF}$} & \multicolumn{2}{|c|}{ Ipsilateral EMG } & \multirow{3}{*}{$\begin{array}{l}85 \\
8.9+\end{array}$} & \multirow{3}{*}{$\begin{array}{l}97 \\
7.9 \dagger\end{array}$} & \multirow{3}{*}{$\begin{array}{l}109 \\
14 \cdot 2+\end{array}$} & \multirow{3}{*}{$\begin{array}{l}121 \\
29 \cdot 7+\end{array}$} & \multirow[b]{2}{*}{$133^{*}$} \\
\hline & $\overline{61}$ & $\overline{7} \overline{3}$ & & & & & \\
\hline & $50.0 \dagger$ & $9 \cdot 3 \dagger$ & & & & & $39 \cdot 5 \dagger$ \\
\hline LP & $56.4 \dagger$ & $50 \cdot 5 \dagger$ & $94 \cdot 5+$ & $61 \cdot 3 \dagger$ & $66 \cdot 4+$ & $64 \cdot 7 \dagger$ & $109 \cdot 5$ \\
\hline DB & $55 \cdot 8 \dagger$ & $41 \cdot 6 \dagger$ & $55 \cdot 5 \dagger$ & $73.0+$ & $64 \cdot 4+$ & $105 \cdot 0$ & $84.9 \dagger$ \\
\hline MB & $44 \cdot 2 \dagger$ & $69 \cdot 2 \dagger$ & $81 \cdot 1 \dagger$ & $71 \cdot 4+$ & $92.8+$ & $85.0 \dagger$ & $96 \cdot 6$ \\
\hline JS & $60 \cdot 3+$ & $56.0+$ & $74 \cdot 7 \dagger$ & $48 \cdot 3 \dagger$ & $84 \cdot 8+$ & $100 \cdot 5$ & $108 \cdot 9$ \\
\hline \multirow[t]{2}{*}{ M } & $53 \cdot 3$ & $45 \cdot 3$ & $62 \cdot 9$ & $52 \cdot 4$ & $64 \cdot 7$ & $77 \cdot 2$ & 87.9 \\
\hline & \multicolumn{7}{|c|}{ Contralateral EMG } \\
\hline & $\overline{61}$ & 73 & 85 & 97 & 109 & 121 & $133^{*}$ \\
\hline JF & $72 \cdot 7 \dagger$ & $21 \cdot 8 \dagger$ & $22 \cdot 7 \dagger$ & $22 \cdot 3 \dagger$ & $22 \cdot 7 \dagger$ & $38.8 \dagger$ & $55.0 \dagger$ \\
\hline LP & $39 \cdot 3+$ & $68 \cdot 0 \dagger$ & 104.9 & $72 \cdot 8 \dagger$ & $69.0+$ & $71.9 \dagger$ & 124.4 \\
\hline DB & $64 \cdot 9+$ & $57 \cdot 7 \dagger$ & $74 \cdot 7 \dagger$ & $96 \cdot 7$ & $87.0 \dagger$ & $96 \cdot 8$ & 90.0 \\
\hline$M B$ & $44.3+$ & $76 \cdot 6 \dagger$ & $97 \cdot 1$ & $85.6 \dagger$ & $82.6+$ & 97.9 & $101 \cdot 6$ \\
\hline JS & $49 \cdot 5 \dagger$ & $58 \cdot 2 \dagger$ & $69 \cdot 7 \dagger$ & $61 \cdot 0 \dagger$ & $80 \cdot 1 \dagger$ & $100 \cdot 0$ & $86 \cdot 6 \dagger$ \\
\hline M & $54 \cdot 1$ & $56 \cdot 5$ & $73 \cdot 8$ & $67 \cdot 7$ & $68 \cdot 3$ & $81 \cdot 1$ & $91 \cdot 7$ \\
\hline
\end{tabular}

*Refers to time of beginning of a $12 \mathrm{~ms}$ summation epoch after onset of reflex shock.

$\uparrow$ Indicates relative EMG activity significantly below control level, $p \leqslant 0.01$. (Pre-shock mean EMG activity set at $100 \%$.)

$81 \%$ of control levels. It should be noted that the resolution of the analysis procedures was limited and failed to illustrate the sometimes greater relative declines in EMG amplitude after R1. For example, inspection of fig 1 (left panel) revealed the near complete cessation of EMG activity after $\mathbf{R} 1$.

The sizes of the ipsilateral and contralateral $\mathrm{R} 2$ responses did not appear to be decreased by the preceding R1 silence but were slightly (but nonsignificantly) greater than the $\mathrm{R} 2$ responses elicited during the relaxation state. There was considerable between-subject variation in the amount of EMG silence after the R2 response. The table presents the relative EMG activity from orbicularis oculi muscles of each individual and the group mean EMG amplitudes from 61 to $144 \mathrm{~ms}$ after onset of the reflex eliciting stimulus. Also of note was the occasional appearance of the R3 response in the silent period (for example, fig 2) during near maximal contractions. This occurred even if $\mathbf{R} 3$ responses were not evoked when the orbicularis oculi muscles were at rest. Thus, the muscle silence that affected the expression of the voluntary contraction had no decremental effect upon subsequent reflex activity.

The grouped $(n=5)$ absolute EMG amplitude data across all intervals pre- and post-stimulus are depicted in fig 3 . When subjects were contracting the eyelid muscles the EMG activity of orbicularis oculi muscles for the group was, of course, greater than at rest. This was observed throughout the complete sampling period from $100 \mathrm{~ms}$ before to $240 \mathrm{~ms}$ after reflex elicitation. 


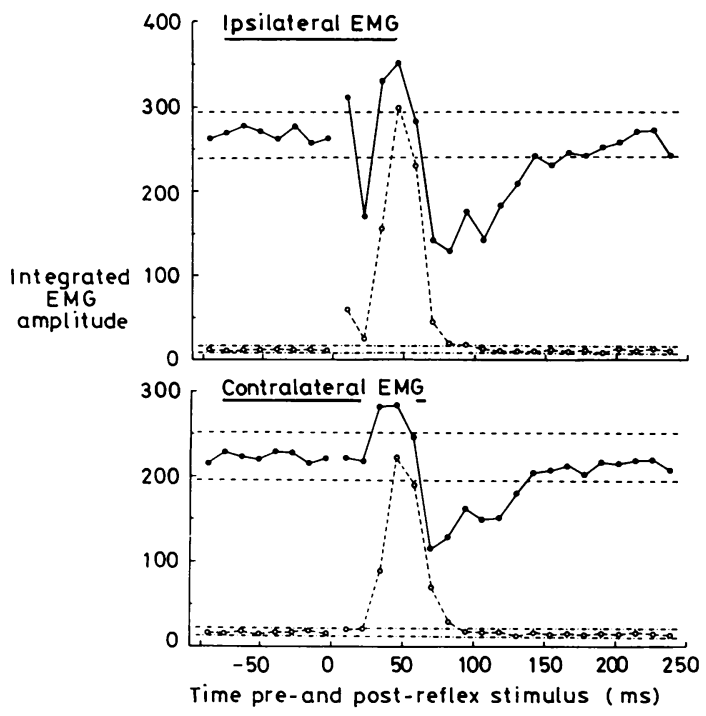

Fig 3 Mean EMG activity for the group during relaxation and contraction of eyelid muscles. Upper panel-ipsilateral EMG. Open circles connected by dashed lines inticates relaxed state, while closed cirsles connected by solid lines indicates contraction state. Lower panel-contralateral EMG. Same symbols as before. Dashed lines indicate the upper and lower boundaries of the $95 \%$ confidence limits constructed about the error term from the analysis of variance. See text for additional description.

Inferential analyses revealed a main effect of contraction state, $\mathrm{F}(1,4)=42 \cdot 76, \mathrm{p} \leqslant 0.005$.

Only minor fluctuations of the averaged EMG activity from the group were observed in the baseline period. In comparison to this relative stability of activity, several changes in summed EMG activity occurred after delivery of the reflex eliciting stimulus: These were related to the presence of R1 (ipsilateral) and R2 (bilateral) and the silent periods that followed each of the responses. For the group, the EMG activity recovered to pre-response baseline levels by about $100 \mathrm{~ms}$ or $200 \mathrm{~ms}$ after reflex elicitation during the relaxed or contraction states, respectively. Statistical analyses indicated that these changes in EMG activity over time were significant, $F(27$, $108)=19 \cdot 42, \quad p \leqslant 0.0001$. Additional analyses showed that there was an interaction between the side of recording and intervals, $\mathrm{F}(27,108)=$ $5.59, \mathrm{p} \leqslant 0.001$, the interaction resulting because of the absence of the early $R 1$ component in the contralateral recording.

One additional finding was that the latencies of both the ipsilateral $\mathrm{R} 1$ and $\mathbf{R} 2$ responses decreased when reflexes were elicited during near maximal contractions ( $\mathrm{R} 1: \mathrm{t}[4]=4 \cdot 08, \mathrm{p}<$ $0.01 ; \mathrm{R} 2 ; \mathrm{t}[4]=3.35, \mathrm{p}<0.05$, two-tailed). The $\mathrm{R} 1$ latency for the group was $13.45 \mathrm{~ms}$ in the muscle relaxation condition and $10.92 \mathrm{~ms}$ during eyelid muscle contractions. For these same conditions, the ipsilateral $\mathrm{R} 2$ response latency was $38.67 \mathrm{~ms}$ and $33.7 \mathrm{~ms}$ in the relaxed and contraction state, respectively.

\section{Discussion}

The major finding of note is that there is a silent period in the eyelid musculature when reflexive activity is elicited in the midst of a voluntary contraction. If it is agreed that the common physiological mechanisms that are presumed responsible for silent periods in limb musculature are not available to orbicularis oculi muscle then other explanations must be sought for the present findings. Some characteristics of the current and other results suggest that the effects occur at levels rresynaptic to the facial motoneuron. First, since the refractory period of facial motoneurons endures for only about 2 to $3 \mathrm{~ms}^{9}$ and subnormal excitability of facial reflexes following conditioning cutaneous stimuli lasts for only about $40 \mathrm{~ms},{ }^{13}$ facial motoneurons would appear to be easily excited after discharge or preliminary tactile stimulation. Secondly, it is important to note that although the volitional EMG activity was depressed, all reflex components appeared to be enhanced when reflexes were elicited during voluntary contractions. Indeed, more systematic experiments have shown that both components of the eyeblink reflex are enhanced for up to $3 \cdot 2 \mathrm{~s}$ after onset of maintained voluntary contraction of orbicularis oculi muscle. ${ }^{21}$ This would suggest that the input from upper motoneurons that mediate volitional activation of facial motoneurons is disrupted either by a gating mechanism activated by reflex stimuli or by the occurrence of the reflex itself. In the peripheral limb musculature this function is usually assigned to the muscle spindle; that is drive from the alpha motoneuron tends to unload the spindle apparatus thereby automatically reducing the amount of spindle drive on the alpha motoneuron and a muscle silent period ensues.

Since there appear to be no muscle spindles in the palpebral musculature ${ }^{1}$ the above mechanisms for muscle silence cannot be invoked. However, a more complicated mechanism utilising cutaneous afferent activity as the source of feedback has been hypothesised in the control of skilled behaviour in the limbs; a similar mechanism may also be involved in the orbicularis 
oculi muscle. The experimental basis of the mechanism is the effect of perturbations on skilled motor performance. When task performance is artificially accelerated voluntary drive is temporarily withdrawn and an effect similar to a silent period is seen. ${ }^{14}$ The latency of the effect, and the effects of anaesthetics upon it suggest that this silent period results partially from cutaneous as well as muscle afferents. ${ }^{15}$ This "silent period" is interpreted not as a segmental effect but as the outcome of a central comparison of obtained feedback with an "expected" feedback based on some sort of corollary discharge $^{26}$; when incoming response-produced stimulation exceeds that level set by the corollary discharge, then the programmed drive onto the motor neuron is reduced. Conceptual schemes of this sort are described by Kelso and Stelmach, ${ }^{8}$ and Phillips and Porter ${ }^{20}$ have described a neural model of cortical and subcortical interaction that could provide a neural substrate for such a scheme. It is apparent that the conceptual mechanisms described by these writers would be sufficient to produce a silent period in orbicularis oculi muscle. That is, the additional burst of reflex activity, summed with that normally present during the voluntary contraction, would necessarily exceed that level programmed with the contraction, and, thus, the drive onto the motoneuron would temporarily diminish. The rapidity of the adjustment and its appearance only after reflex responses in both the ipsilateral and contralateral orbicularis oculi muscles suggests that the hypothetical "comparator mechanism" is in the brainstem, close to the final pathway to the facial motoneurons. Whether these mechanisms operate to generate the silent period in orbicularis oculi is speculative. However, the sensory basis for such comparisons exists as has been shown by the findings that activation of facial and trigeminal cutaneous afferents alters excitability and discharge rates of facial motoneurons. ${ }^{916} 2829$

Why we observed silent periods in orbicularis oculi muscle when other experimenters, using apparently similar methods, did not, is a matter for conjecture. It may be that these reported failures resulted from a choice of stimulus variahles that were not suitable for eliciting vigorous reflex contractions. In fact a careful examination of fig 5 in the Penders and Delwaide report ${ }^{19}$ discloses that there were slight decrements in the voluntary EMG activity following reflex activity. In addition to the possibility that earlier researchers did not use sufficiently intense eliciting stimuli, it has recently been shown that other stimulus variables affect the expression of eyelid reflex activity. If eyeblink reflexes are elicited very soon after the onset of voluntary activity then the R2 component is markedly depressed ${ }^{21}$ as it is also if the eliciting stimuli are presented in a fixed repetitive cycle, ${ }^{422}$ or following a warning signal. ${ }^{7}$ Warning signals may be inadvertently given if the subject and experimenter share the same environment and many earlier experimenters used a relatively uncontrolled setting for their experiments. As noted above, any account of these discrepancies must be speculative, but there can be appreciable diminution in voluntary activity in orbicularis oculi muscles following reflex action in the same.

\section{References}

1 Bowden REM, Mahran ZY. The functional significance of the pattern of innervation of the muscle quadratus labii superious of the rabbit, cat and rat. $J$ Anat 1956; 90:217-27.

2 Falls WM, King JS. The facial motor nucleus of the opossum: Cytology and axosomatic synapse. J Comp Neurol 1976; 167:177-204.

3 Granit R. Receptors and Sensory Perception. New Haven: Yale University Press, 1956.

4 Gregoric M. Habituation of the blink reflex: Role of selective attention. In: Desmedt JE, ed. New developments in electromyography ant clinical neurophysiology. Vol 3. Basel: Karger, 1973: 673-7.

5 Hiraoka M, Shimamura M. Neural mechanisms of the corneal blinking response in cats. Brain Res 1977; 125:265-75.

6 Hufschmidt H-J. The demonstration of autogenetic inhibition and its significance in human voluntary movement. In: Granit R, ed. Nobel svmposium, muscular afferents and motor control. Stockholm: Almqvist and Wiksell, 1966: 269-74.

7 Ison JR, Sanes JN. Conditioned enhancement and diminution of human eveblink reflexes. Bull Psychonom Soc 1979; 14:239.

8 Kelso JA, Stelmach GE. Central and peripheral mechanisms in motor control. In: Stelmach GE, ed. Motor control: Issues and tren's. New York: Academic Press, 1976: 1-49.

9 Kitai ST, Tanaka T, Tsukahara N, Yu H. The facial nucleus of the cat: Antidromic and synpatic activation and peripheral nerve representation. Exp Brain Res 1972; 16:169-91.

10 Kugelberg E. Facial reflexes. Brain 1952; 75: 385-96.

11 Kugelberg E, Eklund K, Grimby L. An electromyographic study of the nociceptive reflexes of the lower limb. Mechanisms of the plantar responses. Brain 1960; 83:393-410.

12 Liberson WT. Monosynaptic reflexes and their clinical significance. Electroenceph Clin Neurophysiol 1962; Supplement. 22:79-89. 
13 Lindquist WT. Analysis of facial reflex facilitation and inhibition by microelectrode recording from the brain stem. Acta Physiol Scan 1972; 85:183-92.

14 Marsden CD, Merton PA, Morton HB. Servo action in the human thumb. $J$ Physiol 1976; 257: 1-44.

15 Marsden CD, Merton PA, Morton HB. The sensory mechanism of servo action in human muscle. J Physiol 1977; 265:521-35.

16 Martin MR, Biscoe TJ. Physiological studies on facial reflexes in the rat. $Q J$ Exp Physiol 1977; 62:209-21.

17 Merton PA. The silent period in a muscle of the human hand. J Physiol 1951; 114:183-98.

18 Ongerboer de Visser BW, Kuypers HGJM. Late blink reflex changes in lateral medullary lesions: An electrophysiological and neuro-anatomical study of Wallenberg's syndrome. Brain 1978; 101:285-94.

19 Penders CA, Delwaide PJ. Physiologic approach to the human blink reflex. In: Desmedt JE, ed. New developments in electromyography and clinical neurophysiology, Volume 3. Basel: Karger, 1973: 649-57.

20 Phillips CG, Porter R. Corticospinal neurons: Their role in movement. New York: Academic Press, 1977.

21 Sanes JN, Ison JR. Voluntary movement and excitability of human cutaneous eyeblink reflexes. Neurosci Abs 1979; 5:385.
22 Sanes JN, Ison JR, Adelson AA. Reflexogenic and psychogenic reflex modulation: Differential effects according to polysynaptic and oligosynaptic pathways to orbicularis oculi in humans. Neurosci Abs 1978; 4:304.

23 Shahani BT. The human blink reflex. J Neurol Neurosurg Psychiatry 1970; 33:792-800.

24 Shahani BT, Young RR. Studies of the normal human silent period. In: Desmedt JE, ed. New developments in electromyography and clinical neurophysiology. Volume 3. Basel: Karger, 1973a: 589-602.

25 Shahani BT, Young RR. Blink reflexes in orbicularis oculi. In: Desmedt JE, ed. New developments in electromyography and clinical neurophysiology. Volume 3. Basel: Karger, 1973b: 641-8.

26 Sperry RW. Neural basis of the spontaneous optokinetic response produced by visual inversion. J Comp Physiol Psychol 1950; 43:482-9.

27 Tanaka T. Synaptic activation of facial afferents on the facial neurons of the cat. Brain Res 1977; 123:378-83.

28 Van Hasselt P. Facial reflexes evoked by electrical stimulation of peripheral facial nerve branches in the cat: Experiments of the auriculotemporal-facial nerve anastomoses. Exp Neurol 1976; 51:407-13.

29 Willer JC, Lamour Y. Electrophysiological evidence for a facio-facial reflex in the facial muscles in man. Brain Res 1977; 119:459-64. 